
\title{
25 Research Square \\ Ultrafast Transient Infrared For Probing Trapping States In Hybrid Perovskite Films
}

\section{Ahmed M. El-Zohry ( $\nabla$ amfzohry@yahoo.com )}

King Abdullah University of Science and Technology (KAUST) https://orcid.org/0000-0003-2901-5815

\section{Bekir Türedir}

King Abdullah University of Science and Technology (KAUST)

\section{Abdullah Alsalloum}

King Abdullah University of Science and Technology (KAUST)

\section{Partha Maity}

King Abdullah University of Science and Technology (KAUST)

\section{Osman M. Bakr}

King Abdullah University of Science and Technology (KAUST)

Boon S. Ooi

King Abdullah University of Science and Technology (KAUST)

\section{Omar F. Mohammed}

King Abdullah University of Science and Technology (KAUST)

\section{Article}

\section{Keywords:}

Posted Date: February 7th, 2022

DOI: https://doi.org/10.21203/rs.3.rs-1315125/v1

License: (c) (1) This work is licensed under a Creative Commons Attribution 4.0 International License. Read Full License

Version of Record: A version of this preprint was published at Communications Chemistry on May 30th, 2022. See the published version at https://doi.org/10.1038/s42004-022-00683-7. 


\section{Abstract}

Studying the charge dynamics of perovskite materials is a crucial step to understand the outstanding performance of these materials in various fields. Herein, we utilize transient absorption in the mid-infrared region, where solely electron signatures in the conduction bands are monitored without external contributions from other dynamical species. Within the measured range of $4000 \mathrm{~nm}$ to $6000 \mathrm{~nm}$ (2500$\left.1666 \mathrm{~cm}^{-1}\right)$, the recombination and the trapping processes of the excited carriers could be easily monitored. Moreover, we reveal that within this spectral region the trapping process could be distinguished from recombination process, in which the iodide-based films show more tendencies to trap the excited electrons in comparison to the bromide-based derivatives. The trapping process was assigned due to the emission released in the mid-infrared region, while the traditional band-gap recombination process did not show such process. Various parameters have been tested such as film composition, excitation dependence and the probing wavelength. This study open new frontiers for the transient midinfrared absorption to assign the trapping process in perovskite films both qualitatively and quantitatively.

\section{Introduction:}

Hybrid perovskite materials have recently attracted lots of attention due to their unique photo-physical properties and their high performances in various applications such as solar cells and light emitting diodes. ${ }^{1-13}$ However, still controlling the amount of traps present in these materials especially upon making thin films is a challenging procedure. ${ }^{14-15}$ The presence of trap states either through structural defects or other types can quench the charge carriers motilities inside the materials and thus reducing both the device's performance and its stability. $8,14,16-18$ Various direct and indirect methods have been applied to track and quantify trap states such as electrical or optical measurements, however, most of these still have some drawbacks. ${ }^{8,14-19}$ For instance, conductivity measurements have low time resolutions and can't distinguish between various carriers such as electrons and holes especially upon having close motilities. ${ }^{20}$ Also, the most commonly used optical measurements such as time-resolved photoluminescence or transient absorption in the visible range couldn't afford direct spectral signatures for trapping states except providing variations of multi-exponential kinetic rates between different samples according to the estimated traps present in the investigated samples. ${ }^{1-2,7,13,21-23}$ Thus, still there is a need for a direct transient optical method to track and quantify the trapping process in perovskite materials, and correlate their presence directly to charge dynamics. For that sake, we utilized mid-infrared (mid-IR) probe to monitor the charge dynamics of four different perovskite films with different compositions. Following the charge dynamics using femtosecond transient absorption (fs-TA) in the mid-IR has been used previously for several systems including metal complexes ${ }^{24-27}$, organic dyes $^{28-33}$, metals ${ }^{34}$, and semiconductors ${ }^{17,} 20,35-39$.

Basically for semiconductors, the electron's absorption in the conduction band with high density of states has a broad spectral signature extending from $3,333 \mathrm{~nm}\left(3000 \mathrm{~cm}^{-1}\right)$ to $11,111 \mathrm{~nm}\left(900 \mathrm{~cm}^{-1}\right)$, in which 
other contributions from cationic or anionic molecular species present can be easily quantified. ${ }^{20,24,28-29}$ The positive signature in the mid-IR upon photon excitation is ascribed to the presence of intra-transition of free electrons in/into the conduction band of the semiconductor used. ${ }^{20,39-40}$ The transient mid-IR was used to follow trapped electrons at the mid-gap shallow states in the platinized $\mathrm{TiO}_{2}$ system, in which IR emission (heat) is evolved in the IR region as a result of electron trapping process. ${ }^{20,39}$ Recent mid-IR studies have been done on perovskite materials, however in those studies the authors focused on following the $\mathrm{NH}$ vibrational modes, present in the organic cationic part of the perovskites. ${ }^{16-17,21,41}$ In contrast, in our selected mid-IR region, we don't have any contribution of the vibrational modes of the organic part, only transient signal of electrons in the conduction band, see Figure S1.

Herein, we propose using fs-TA in the mid-IR region as a sensitive tool to follow the presence of traps in hybrid perovskite films. In the current study, various hybrid perovskite films have been synthesized and utilized to study the charge dynamics in the mid-IR region extending from ca. 4000-6000 nm (2500-1666 $\mathrm{cm}^{-1}$ ). We found that perovskite films with methyl-ammonium cation (MA) and halides with lodide (I) derivatives tend to emit mid-IR than other films with formamidinium (FA) and bromide ( $\mathrm{Br}$ ) derivatives, depending on the working conditions and the quality of the prepared films (see steady state measurements in Figure S2-4). Thus, we present herein the fs-TA in the mid-IR as a potential transient optical tool towards deeper understandings of charge dynamics in perovskite films.

\section{Results And Discussions:}

Figure $1 \mathrm{~A}$ shows the false 2-D plot fs-TA in the mid-IR region with a central detection window of $5000 \mathrm{~nm}$ $\left(2000 \mathrm{~cm}^{-1}\right)$ for $\mathrm{MAPbBr}_{3}$ thin film using excitation wavelength of $530 \mathrm{~nm}$. At time zero, an intense positive signal appears due to the population of electrons in the conduction band. ${ }^{24,28,39,42}$ This transient positive signal decays exponentially towards zero within few nanoseconds. However, the extracted spectra show negative features at longer time scale $>1.0 \mathrm{~ns}$; see Figure 1B. Also, the extracted kinetic trace at $4900 \mathrm{~nm}$ shows a multi-exponential decay for the positive signal with an average lifetime of 120 ps, followed by a small negative feature beyond 2 ns; see Figure $1 \mathrm{C}$.

The same film ( $\left.\mathrm{MAPbBr}_{3}\right)$ was measured by fs-TA in the visible range, and an extracted kinetic trace at $550 \mathrm{~nm}$ corresponding to the ground state bleach (GSA) is compared with the extracted kinetic trace at $4900 \mathrm{~nm}$ from the mid-IR region; see Figure 1D and Figure S5. The comparison shows that both normalized kinetic traces from different spectral regions are very similar, except the presence of a new feature at the extracted kinetic trace from the mid-IR range; see Figure 1C-D. The similarity between the two kinetic traces for $\mathrm{MAPbBr}_{3}$ film confirms the validity of mid-IR signal to trace charge dynamics in perovskite films. However, the charge dynamics at in the mid-IR region are not similar. For example, negative features at the red-part of the false $2 \mathrm{D}$ plot $(4900 \mathrm{~nm})$ in Figure 1A, appears differently than in the blue-part $(5120 \mathrm{~nm})$. Extracting a kinetic trace at $5120 \mathrm{~nm}\left(1953 \mathrm{~cm}^{-1}\right)$ shows earlier conversion of positive to negative signals at ca. 200 ps; see Figure 1C. Also, comparing this kinetic trace with the one 
extracted from the GSB in the visible region, shows different behavior than kinetic trace at $4900 \mathrm{~nm}$; see Figure 1D. This highlights the dependence of such negative feature on the probed window.

Upon measuring the iodide-derivative, $\mathrm{MAPbl}_{3}$ film, a detectable fs-TA mid-IR signal was also found, but with different behavior, see Figure 2A-B. Interestingly, the transient mid-IR signal for $\mathrm{MAPbl}_{3}$ film was changing over minutes time scale; see Figure $2 \mathrm{C}$. Thus, various kinetic traces were extracted at different times and compared together at the same proved wavelength. For instance, the fresh-irradiated film (0 min.), appositive signal was measured until ca. 100 ps, and then a small negative signal started to emerge. The disappearance of the positive signal became faster with the longer the exposure process associated with an increase of the signal at early times; see Figure 2C. For example, at 26 minute of irradiation, the positive signal converted into a negative signal within 10 ps; see Figure $2 \mathrm{C}$. Interestingly, this process is reversible, in which switching off the irradiation for almost 8 minutes, and re-measure the dynamics again at the same irradiated spot (@34 minutes in the Figure 2C), the dynamics slowly started to be similar to the 10 minutes measurements; see Figure $2 \mathrm{C}$. Moreover, after the appearance of the negative signal it decays later on to zero due to the recombination process, see Figure $2 \mathrm{C}$.

Upon measuring the same film in the visible range, a strong GSB signal at ca. $760 \mathrm{~nm}$ was observed overlapping with an ESA spectra extending from 650 to $850 \mathrm{~nm}$, see Figure S7. However, no unique change in dynamics has been observed in the visible range similar to the shown data in the mid-IR range. And upon comparing the extracted normalized visible kinetic trace at $760 \mathrm{~nm}$ with the ones from the mid$I R$, it is evident the visible kinetic trace is similar to the one from mid-IR at 26 min only at early times; see Figure 2D. However, still at later times, the mid-IR signal switches its sign, but not the visible kinetic trace at $760 \mathrm{~nm}$. The change of charge dynamics upon irradiation has been assigned in iodide-rich perovskite materials to halide-defects assisted by the low energy needed for defects formation. ${ }^{14,43-45}$

This comparison highlights that this appearance of negative features in the mid-IR region is a dynamical process and also reversible ( $\mathrm{MAPbl}_{3}$ case). Additionally, the extracted kinetic trace at $4900 \mathrm{~nm}$ from the $\mathrm{MAPbl}_{3}$ shows stronger and earlier negative signal than the one shown in $\mathrm{MAPbBr}_{3}$ case. Moreover, for further confirmation of the working conditions, a reference silicon wafer substrate was measured under the same procedure, and no negative features have been detected, see Figure S8. Thus, we assign these strong negative signatures in the mid-IR for $\mathrm{MAPbl}_{3}$ film to the emissive trapping process near the VB of perovskites, in which the mid-IR signal is more sensitive than in the visible range. Several studies showed that iodide based perovskites are more vulnerable for formation of more trap states than bromide ones. ${ }^{1}$ $8,14,23$

To scrutinize our interpretation about the sensitivity of mid-IR towards trapping process, we performed the same measurements on other perovskite films including $\mathrm{FAPbBr}_{3}, \mathrm{FAPbl}_{3}$ and mixture of their halides. For the $\mathrm{FAPbBr}_{3}$ film, the mid-IR signal shows primarily a strong negative signal close to time zero converting into a positive signal with a lifetime of ca. $60 \mathrm{fs}$, which has been assigned to the exciton thermalization/dissociation process ${ }^{7}$; see Figure 3A-B. Previously, in the $\mathrm{MAPbBr}_{3}$ film, exciton binding 
energy seems to be smaller, thus, no detection of exciton dissociation process could be seen; see Figure 1. Then the charge recombination in the $\mathrm{FAPbBr}_{3}$ film (decay of the TA signal) has been fitted with multi exponential behavior, giving an average lifetime is about 10 ps, see Figure 3B.

For the $\mathrm{FAPbl}_{3}$ film, similar observation was estimated for the lifetime needed for exciton dissociation in $\mathrm{FAPbl}_{3}$ film; see Figure 3C-D. However, instead of charge recombination, the signal converted again to a negative signal due to a trapping process of time component ca. $8.5 \mathrm{ps}$; see Figure 3D. Then the trapped electrons recombine slowly with a lifetime higher than $1 \mathrm{~ns}$. It is clear that this negative signature in the mid-IR range is associated with the iodide derivative of hybrid perovskite films due to the presence of trapping centers in the iodide perovskites more than in bromide perovskites.

To verify the role of iodide anion for the formation of emissive trapping centers, we synthesized other set of various perovskite films of different ratios between the iodide and bromide halides $\left(\mathrm{MAPbl}_{n} \mathrm{Br}_{3-n}\right)$ to investigate the effect of doping with iodide ions on the presence of such negative signal. Figure $4 \mathrm{~A}$ shows the kinetic traces of mid-IR signals for four films of $\mathrm{MAPbI}_{n} \mathrm{Br}_{3-n}$, in which $\mathrm{n}$ varies from 0 to 3 . The extracted kinetics at $4900 \mathrm{~nm}$ show the appearance of negative signals at different times ranging from 100 ps to $1 \mathrm{~ns}$ depending on the amount of iodide halide present, in which higher content of iodide shows faster appearance of negative mid-IR signal. Thus, the iodide content in perovskite films controls the appearance of the negative signal (emission of mid-IR).

To study the dependence of appearance of these negative signals on the energy of the mid-IR probe, the $\mathrm{MAPbl}_{3}$ film is excited at $520 \mathrm{~nm}(300 \mu \mathrm{W})$, and probed at various mid-IR energy ranging from $4000 \mathrm{~nm}$ to $6000 \mathrm{~nm}$, as shown in Figure 4B. As expected, the appearance time of the negative signal depends on the utilized mid-IR energy, in which the switching points from positive to negative signal happen at ca. $500 \mathrm{ps}$ when using $4000 \mathrm{~nm}$ and at ca. $20 \mathrm{ps}$ upon using $6000 \mathrm{~nm}$. This also illustrates the incapability of visible probe light to directly track the trapping process due the high energy carried by the photons used.

Furthermore, by changing the excitation wavelength from $410 \mathrm{~nm}$ to $520 \mathrm{~nm}$ for another $\mathrm{MAPbl}_{3}$ film, the extracted kinetic traces at $4500 \mathrm{~nm}$ show a dependence of the kinetic decay with the wavelength used; see Figure 4C. The appearance of negative signal is faster upon using $410 \mathrm{~nm}$ then became slower with $440 \mathrm{~nm}$, and $520 \mathrm{~nm}$ respectively. Upon using the excitation light at $520 \mathrm{~nm}$, the kinetic trace decay to zero with no signature of negative signal, despite that higher power was used, almost 20 times higher $(300 \mu \mathrm{W})$ than in $410 \mathrm{~nm}$; see Figure $4 \mathrm{C}$. This excitation energy dependence shows that the higher the electron can be promoted in the excited state, the higher than chances to be trapped.

From the above mid-IR transient measurements, the following mechanism can be drawn, see Scheme 1 . Upon exciting the perovskite film, the electron in the CB should absorb the following mid-IR probe to populate various vibrational levels in the excited state, giving a positive transient absorption signal. Due to the presence of trap states within the bandgap of the perovskite film, the electron in the CB decays nonradiatively to the ground state, in which the transient signal decays to zero. Interestingly, upon using synthesizing perovskite films using chemical species such as $\mathrm{MA}^{+}$or l- or both of them as in $\mathrm{MAPbl}_{3}$, 
negative transient signal in the mid-IR region starts to appear. And since perovskite films have no characteristic features in this mid-IR region, this negative transient signal can be only due to emission of mid-IR signal. It is already established in the literature that MA/iodide perovskite films show more potential to form trap states than other derivatives ( $\mathrm{FA} / \mathrm{Br}$ ). ${ }^{1,23}$ Thus, we postulate that in $\mathrm{MAPbl}_{3}$ films, different kind of emissive trap states are additionally formed related to ion migration and new phonon modes in these films that are not present for instance in the $\mathrm{FAPbBr}_{3}$ films. ${ }^{1,23,46}$

Interestingly, to detect these mid-IR emissive states suitable probe energy should utilized as shown in Figure 4B. These data presents the influence of the energy carried by the probing photons to free the trapped electrons if sufficient energy is present. For instance, the probed pulse at $4000 \mathrm{~nm}\left(2500 \mathrm{~cm}^{-1}\right)$ can liberate the trapped electron more efficient than at $6000 \mathrm{~nm}\left(1666 \mathrm{~cm}^{-1}\right)$, and the appearance of negative signal upon using $4000 \mathrm{~nm}\left(2500 \mathrm{~cm}^{-1}\right)$ will not appear unless the electron is deeply trapped. In the same way, the appearance of negative signal at $6000 \mathrm{~nm}\left(1666 \mathrm{~cm}^{-1}\right)$ is much faster due to lower energy carried by the probe pulse to liberate the trapped electron in the emissive states, allowing for deactivation through the other non-emissive states. These observations are consistent with a previously proposed mechanism that trapped electrons can be excited thermally if the energy difference between the trap state and the $\mathrm{CB}$ is small, $<50 \mathrm{meV} .{ }^{20}$ This also illustrates the incapability of transient absorption in the visible region to detect such a trapping process due to the higher energy carried by the visible probed light, that have the potential to liberate the trapped carriers into higher excited state, producing undistinguishable signal for the trapping process in the visible region.

Moreover, upon using higher band-gap excitations such as $410 \mathrm{~nm}$, the probability of electron trapping in these emissive states is increased despite the excitation intensity used, matching with the expected distribution for the states of trap-density present. We also show that continuous irradiation at high excitation energy for the $\mathrm{MAPbl}_{3}$ film (Figure 2C) increases the rate of trapping (channel 2 in Scheme 1), as well as the intensity of the transient signal. This indicates towards the exciting correlation between the light irradiation and ion migration process in perovskite films. ${ }^{47}$ This means that changes in the perovskite lattice by the incident light (depending on energy) can lead to the formation of these emissive states.

\section{Conclusion:}

We show herein for the first time that transient absorption in the mid-IR region is a suitable tool to explore trapping process in addition to follow the behavior of free electrons in the conduction band without other contributions of other species (reduced/oxidized species). Significantly, the selected region of the mid-IR spectrum can be utilized to follow the actual trapping process of electrons by detecting the negative appearance of the transient signal, which is likely due to the formation of emissive trap states. We also figure out that similar measurements in the visible region could not be monitored due the energy of the probed light that can liberate the trapped carriers into higher excitation levels, providing additional complexity to distinct between tarped carriers and other species such as excitons and free carriers 
absorption. Interestingly, these emissive trap states in the mid-IR can be controlled by film quality, film chemical composition, and utilized band-gap excitation energy. This work will open frontiers towards understanding and controlling the nature of trapping centers in perovskite films.

\section{References:}

1. Alarousu, E.; El-Zohry, A. M.; Yin, J.; Zhumekenov, A. A.; Yang, C.; Alhabshi, E.; Gereige, I.; AlSaggaf, A.; Malko, A. V.; Bakr, O. M.; Mohammed, O. F., Ultralong Radiative States in Hybrid Perovskite Crystals: Compositions for Submillimeter Diffusion Lengths. JPCL 2017, 8, 4386-4390.

2. Guo, Z.; Wan, Y.; Yang, M. J.; Snaider, J.; Zhu, K.; Huang, L. B., Long-Range Hot-Carrier Transport in Hybrid Perovskites Visualized by Ultrafast Microscopy. Science 2017, 356, 59-62.

3. Zuo, Z. Y.; Ding, J. X.; Zhao, Y.; Du, S. J.; Li, Y. F.; Zhan, X. Y.; Cui, H. Z., Enhanced Optoelectronic Performance on the (110) Lattice Plane of an $\mathrm{Mapbbr}_{3}$ Single Crystal. J. Phys. Chem. Lett. 2017, 8, 684-689.

4. Bi, D., et al., Efficient Luminescent Solar Cells Based on Tailored Mixed-Cation Perovskites. Science Advances 2016, 2.

5. Kadro, J. M.; Pellet, N.; Giordano, F.; Ulianov, A.; Muntener, O.; Maier, J.; Gratzel, M.; Hagfeldt, A., Proofof-Concept for Facile Perovskite Solar Cell Recycling. Energ Environ Sci 2016.

6. Colella, S.; Mazzeo, M.; Rizzo, A.; Gigli, G.; Listorti, A., The Bright Side of Perovskites. JPCL 2016, 7, 4322-4334.

7. Zhai, Y. X.; Sheng, C. X.; Zhang, C.; Vardeny, Z. V., Ultrafast Spectroscopy of Photoexcitations in Organometal Trihalide Perovskites. Adv. Funct. Mater. 2016, 26, 1617-1627.

8. Shi, D.; Adinolfi, V.; Comin, R.; Yuan, M.; Alarousu, E.; Buin, A.; Chen, Y.; Hoogland, S.; Rothenberger, A.; Katsiev, K., Low Trap-State Density and Long Carrier Diffusion in Organolead Trihalide Perovskite Single Crystals. Science 2015, 347, 519-522.

9. Huang, J.; Shao, Y.; Dong, Q., Organometal Trihalide Perovskite Single Crystals: A Next Wave of Materials for 25\% Efficiency Photovoltaics and Applications Beyond? JPCL 2015, 6, 3218-3227.

10. Fang, H. H.; Raissa, R.; Abdu-Aguye, M.; Adjokatse, S.; Blake, G. R.; Even, J.; Loi, M. A., Photophysics of Organic-Inorganic Hybrid Lead lodide Perovskite Single Crystals. Adv. Funct. Mater. 2015, 25, 23782385.

11. Green, M. A.; Bein, T., Photovoltaics Perovskite Cells Charge Forward. Nature Materials 2015, 14, 559-561.

12. Gao, P.; Gratzel, M.; Nazeeruddin, M. K., Organohalide Lead Perovskites for Photovoltaic Applications. Energ Environ Sci 2014, 7, 2448-2463.

13. Stranks, S. D.; Eperon, G. E.; Grancini, G.; Menelaou, C.; Alcocer, M. J. P.; Leijtens, T.; Herz, L. M.; Petrozza, A.; Snaith, H. J., Electron-Hole Diffusion Lengths Exceeding 1 Micrometer in an Organometal Trihalide Perovskite Absorber. Science 2013, 342, 341-344. 
14. Meggiolaro, D.; Motti, S. G.; Mosconi, E.; Barker, A. J.; Ball, J.; Perini, C. A. R.; Deschler, F.; Petrozza, A.; De Angelis, F., lodine Chemistry Determines the Defect Tolerance of Lead-Halide Perovskites. Energ Environ Sci 2018, 11, 702-713.

15. Fang, H.-H.; Adjokatse, S.; Wei, H.; Yang, J.; Blake, G. R.; Huang, J.; Even, J.; Loi, M. A., Ultrahigh Sensitivity of Methylammonium Lead Tribromide Perovskite Single Crystals to Environmental Gases. Science Advances 2016, 2.

16. Munson, K. T.; Kennehan, E. R.; Doucette, G. S.; Asbury, J. B., Dynamic Disorder Dominates Delocalization, Transport, and Recombination in Halide Perovskites. Chem 2018, 4, 2826-2843.

17. Munson, K. T.; Grieco, C.; Kennehan, E. R.; Stewart, R. J.; Asbury, J. B., Time-Resolved Infrared Spectroscopy Directly Probes Free and Trapped Carriers in Organo-Halide Perovskites. ACS Energy Lett. 2017, 2, 651-658.

18. Leblebici, S. Y., et al., Facet-Dependent Photovoltaic Efficiency Variations in Single Grains of Hybrid Halide Perovskite. Nature Energy 2016, 1.

19. Simpson, M. J.; Doughty, B.; Yang, B.; Xiao, K.; Ma, Y.-Z., Imaging Electronic Trap States in Perovskite Thin Films with Combined Fluorescence and Femtosecond Transient Absorption Microscopy. JPCL 2016, 7, 1725-1731.

20. Yamakata, A.; Ishibashi, T.; Onishi, H., Time-Resolved Infrared Absorption Spectroscopy of Photogenerated Electrons in Platinized Tio2 Particles. Chem. Phys. Lett. 2001, 333, 271-277.

21. Guo, P. J., et al., Infrared-Pump Electronic-Probe of Methylammonium Lead lodide Reveals Electronically Decoupled Organic and Inorganic Sublattices. Nat. Commun. 2019, 10.

22. Yin, J.; Yang, H.; Song, K.; El-Zohry, A. M.; Han, Y.; Bakr, O. M.; Bredas, J. L.; Mohammed, O. F., Point Defects and Green Emission in Zero-Dimensional Perovskites. J. Phys. Chem. Lett. 2018, 9, 54905495.

23. Sarmah, S. P., et al., Double Charged Surface Layers in Lead Halide Perovskite Crystals. Nano Lett 2017, 17, 2021-2027.

24. Abdellah, M.; El-Zohry, A. M.; Antila, L. J.; Windle, C. D.; Reisner, E.; Hammarström, L., Time-Resolved Ir Spectroscopy Reveals a Mechanism with Tio2 as a Reversible Electron Acceptor in a Tio2-Re Catalyst System for Co2 Photoreduction. J. Am. Chem. Soc. 2017, 139, 1226-1232.

25. Asbury, J. B.; Ellingson, R. J.; Ghosh, H. N.; Ferrere, S.; Nozik, A. J.; Lian, T. Q., Femtosecond Ir Study of Excited-State Relaxation and Electron-Injection Dynamics of Ru(Dcbpy)(2)(Ncs)(2) in Solution and on Nanocrystalline Tio2 and Al2o3 Thin Films. J. Phys. Chem. B 1999, 103, 3110-3119.

26. Furube, A.; Murai, M.; Watanabe, S.; Hara, K.; Katoh, R.; Tachiya, M., Near-Ir Transient Absorption Study on Ultrafast Electron-Injection Dynamics from a Ru-Complex Dye into Nanocrystalline In2o3 Thin Films: Comparison with Sno2, Zno, and Tio2 Films. J Photoch Photobio A 2006, 182, 273-279.

27. Ellingson, R. J.; Asbury, J. B.; Ferrere, S.; Ghosh, H. N.; Sprague, J. R.; Lian, T. Q.; Nozik, A. J., Dynamics of Electron Injection in Nanocrystalline Titanium Dioxide Films Sensitized with [Ru(4,4 '-Dicarboxy-2,2 '-Bipyridine)(2)(Ncs)(2)] by Infrared Transient Absorption. J. Phys. Chem. B 1998, 102, 6455-6458. 
28. El-Zohry, A. M., The Origin of Slow Electron Injection Rates for Indoline Dyes Used in Dye-Sensitized Solar Cells. Dyes Pigm. 2019, 160, 671-674.

29. El-Zohry, A. M.; Karlsson, M., Gigantic Relevance of Twisted Intramolecular Charge Transfer for Organic Dyes Used in Solar Cells. J. Phys. Chem. C 2018, 122, 23998-24003.

30. Juozapavicius, M.; Kaucikas, M.; Dimitrov, S. D.; Barnes, P. R. F.; van Thor, J. J.; O'Regan, B. C., Evidence for "Slow" Electron Injection in Commercially Relevant Dye-Sensitized Solar Cells by Vis-Nir and Ir Pump-Probe Spectroscopy. J. Phys. Chem. C 2013, 117, 25317-25324.

31. Juozapavicius, M.; Kaucikas, M.; van Thor, J. J.; O’Regan, B. C., Observation of Multiexponential Picoto Subnanosecond Electron Injection in Optimized Dye-Sensitized Solar Cells with Visible-Pump MidInfrared-Probe Transient Absorption Spectroscopy. J. Phys. Chem. C 2012, 117, 116-123.

32. Ghosh, H. N.; Asbury, J. B.; Lian, T. Q., Direct Observation of Ultrafast Electron Injection from Coumarin 343 to Tio2 Nanoparticles by Femtosecond Infrared Spectroscopy. J. Phys. Chem. B 1998, $102,6482-6486$.

33. El-Zohry, A. M.; Zietz, B., Electron Dynamics in Dye-Sensitized Solar Cells Influenced by DyeElectrolyte Complexation. J. Phys. Chem. C 2020, 124, 16300-16307.

34. Furube, A.; Du, L.; Hara, K.; Katoh, R.; Tachiya, M., Ultrafast Plasmon-Induced Electron Transfer from Gold Nanodots into Tio2 Nanoparticles. J. Am. Chem. Soc. 2007, 129, 14852-14855.

35. Pavliuk, M. V.; Fernandes, D. L. A.; El-Zohry, A. M.; Abdellah, M.; Nedelcu, G.; Kovalenko, M. V.; Sa, J., Magnetic Manipulation of Spontaneous Emission from Inorganic Cspbbr3 Perovskites Nanocrystals. Advanced Optical Materials 2016, 4, 2004-2008.

36. Cieslak, A. M., et al., Ultra Long-Lived Electron-Hole Separation within Water-Soluble Colloidal Zno Nanocrystals: Prospective Applications for Solar Energy Production. Nano Energy 2016, 30, 187192.

37. Harrick, N. J., Lifetime Measurements of Excess Carriers in Semiconductors. J. Appl. Phys. 1956, 27, 1439-1442.

38. Tang, J., et al., Colloidal-Quantum-Dot Photovoltaics Using Atomic-Ligand Passivation. Nature Materials 2011, 10, 765-771.

39. Tamaki, Y.; Furube, A.; Katoh, R.; Murai, M.; Hara, K.; Arakawa, H.; Tachiya, M., Trapping Dynamics of Electrons and Holes in a Nanocrystalline Tio2 Film Revealed by Femtosecond Visible/near-Infrared Transient Absorption Spectroscopy. Comptes Rendus Chimie 2006, 9, 268-274.

40. Heimer, T. A.; Heilweil, E. J., Direct Time-Resolved Infrared Measurement of Electron Injection in DyeSensitized Titanium Dioxide Films. J. Phys. Chem. B 1997, 101, 10990-10993.

41. Guo, P. J., et al., Slow Thermal Equilibration in Methylammonium Lead lodide Revealed by Transient Mid-Infrared Spectroscopy. Nat. Commun. 2018, 9.

42. El-Zohry, A. M.; Cong, J.; Karlsson, M.; Kloo, L.; Zietz, B., Ferrocene as a Rapid Charge Regenerator in Dye-Sensitized Solar Cells. Dyes Pigm. 2016, 132, 360-368. 
43. Barker, A. J., et al., Defect-Assisted Photoinduced Halide Segregation in Mixed-Halide Perovskite Thin Films. ACS Energy Lett. 2017, 2, 1416-1424.

44. Samu, G. F.; Janáky, C.; Kamat, P. V., A Victim of Halide lon Segregation. How Light Soaking Affects Solar Cell Performance of Mixed Halide Lead Perovskites. ACS Energy Lett. 2017, 2, 1860-1861.

45. Chen, Y. H., et al., Impacts of Alkaline on the Defects Property and Crystallization Kinetics in Perovskite Solar Cells. Nat. Commun. 2019, 10.

46. Ghosh, S.; Pal, S. K.; Karki, K. J.; Pullerits, T., Ion Migration Heals Trapping Centers in Ch3nh3pbbr3 Perovskite. ACS Energy Lett. 2017, 2, 2133-2139.

47. Kamat, P. V.; Kuno, M., Halide lon Migration in Perovskite Nanocrystals and Nanostructures. Acc. Chem. Res. 2021, 54, 520-531.

\section{Scheme:}

Scheme 1 is available in the Supplemental Files section

\section{Figures}



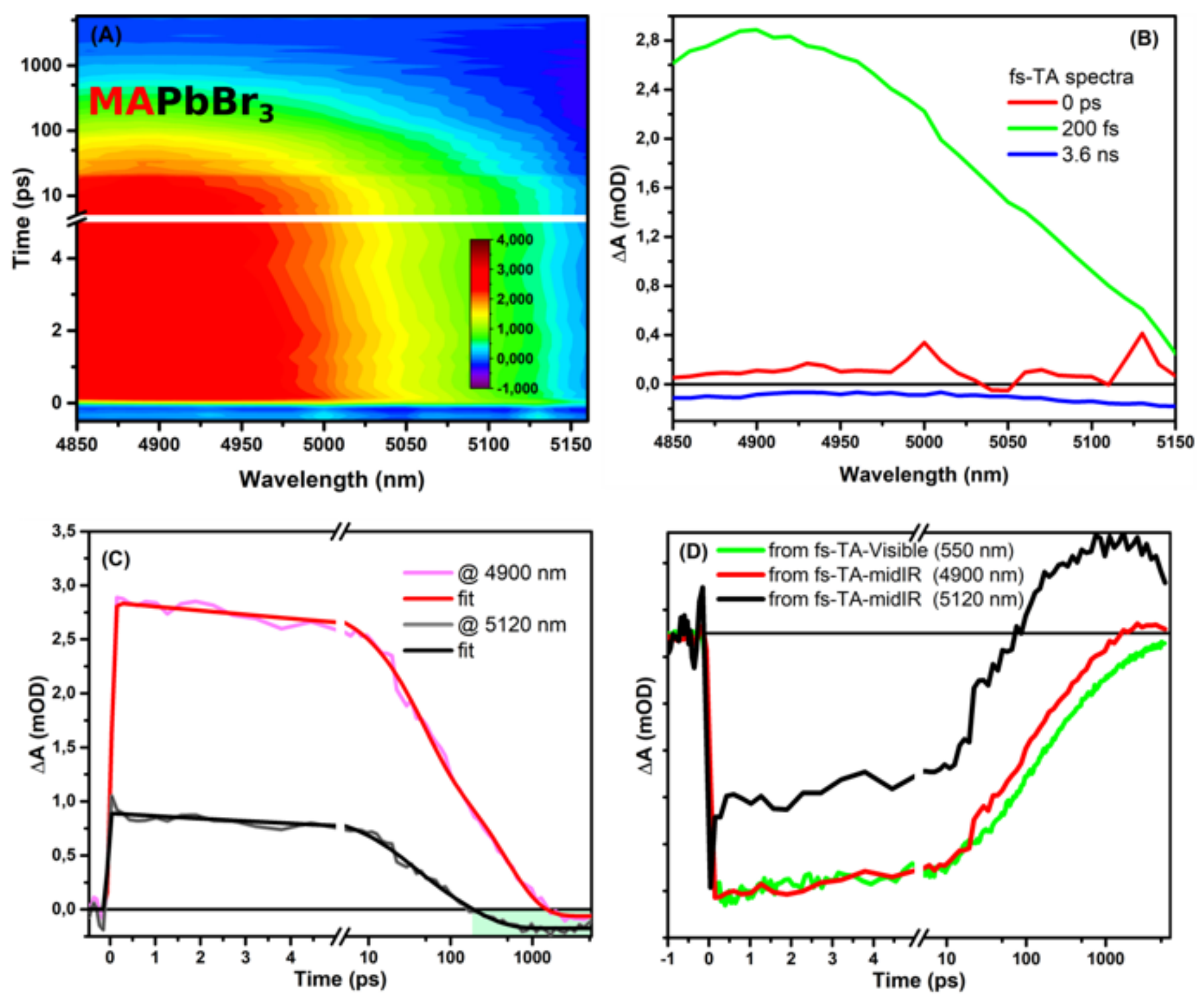

Figure 1

(A) 2D-false color plot for fs-transient absorption in the mid-infrared regions for $\mathrm{MAPbBr}_{3}$ film using 530 $\mathrm{nm}$ as an excitation source. (B) Extracted spectra for transient spectra in mid-infrared region, the spectra are corrected for the wavelength scale. (C) Extracted kinetic trace at 4900 and $5120 \mathrm{~nm}$ with corresponding fitting, green shaded area highlights the appearance of negative signal. (D) Comparison between normalized kinetic traces in the infrared and visible ranges for $\mathrm{MAPbBr}_{3}$ film. 

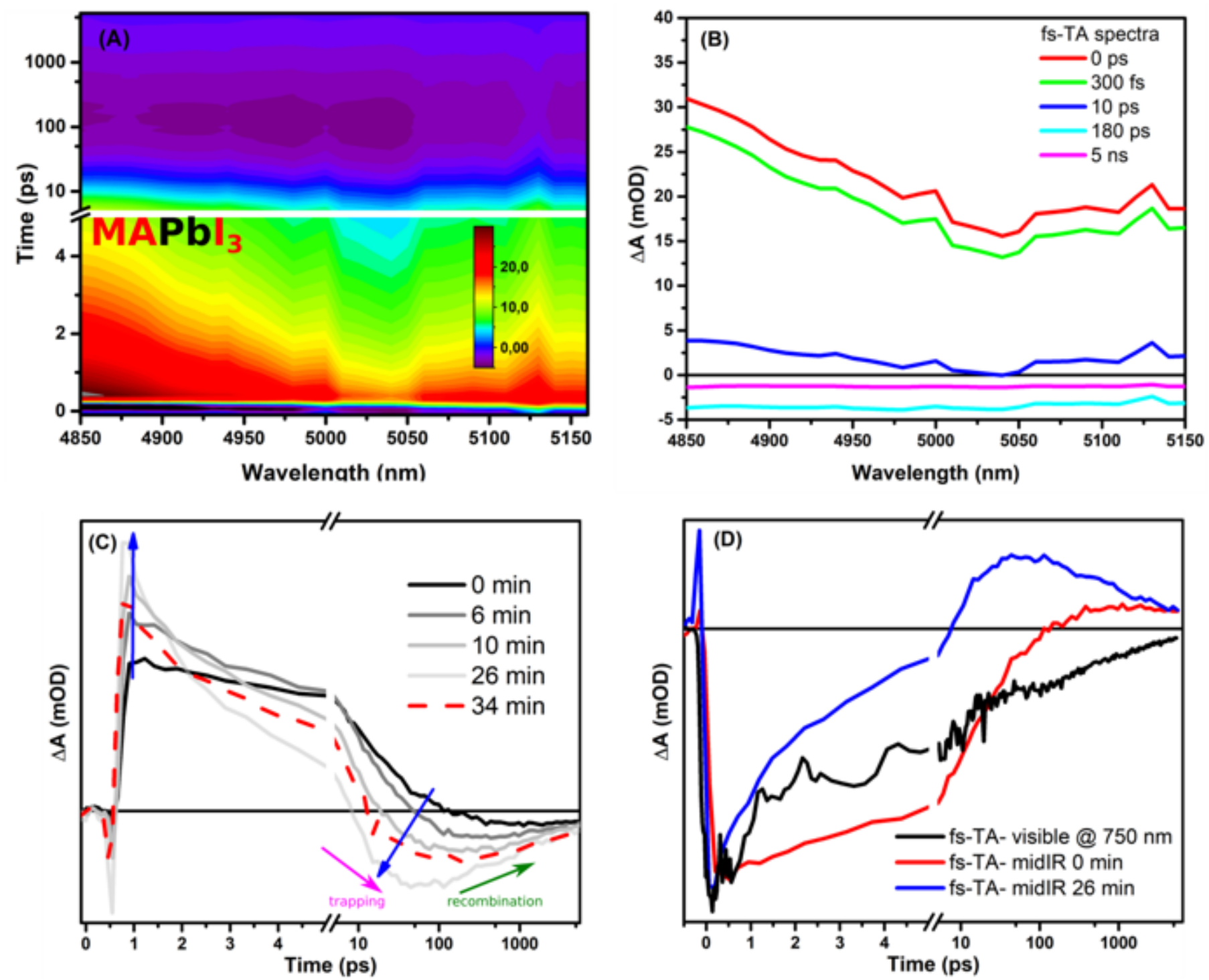

Figure 2

(A) 2D-false color plot for fs-transient absorption in the mid-infrared regions for $\mathrm{MAPbl}_{3}$ film using 530 $\mathrm{nm}$ as an excitation source after 26 minutes of irradiation. (B) Extracted spectra for transient spectra in mid-infrared region (A), the spectra are corrected for the wavelength scale. (C) Extracted kinetic trace at $4900 \mathrm{~nm}$ at different irradiation time shown in minutes for $\mathrm{MAPbl}_{3}$ film, using $520 \mathrm{~nm}$ with excitation power of $500 \mu \mathrm{W}$. (D) Comparison between normalized kinetic traces in the infrared and visible ranges for $\mathrm{MAPbl}_{3}$ film. 

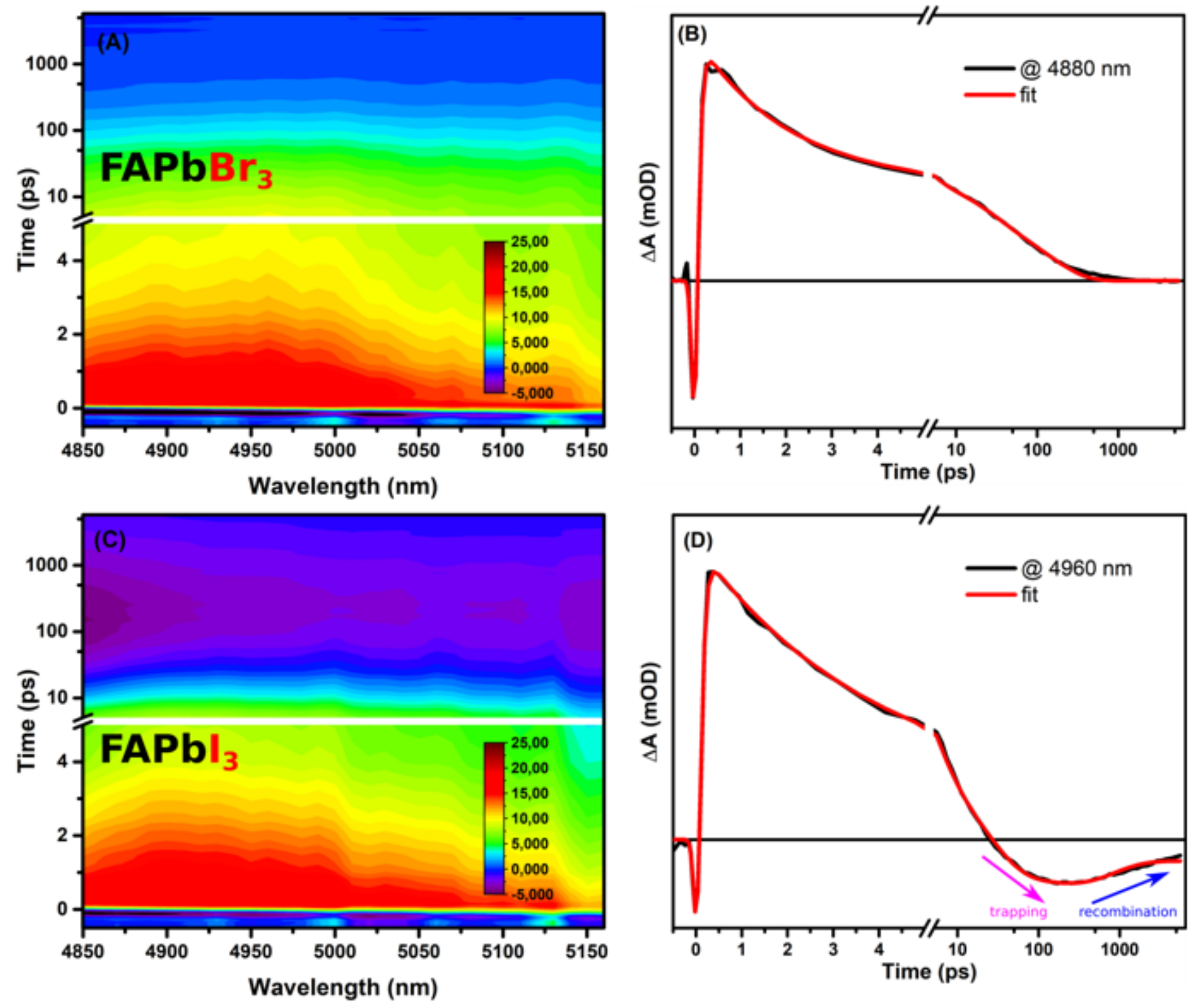

\section{Figure 3}

(A) 2D-false color plot for fs-transient absorption in the mid-infrared regions for $\mathrm{FAPbBr}_{3}$ film using 530 $\mathrm{nm}$ as an excitation source. (B) Extracted kinetic trace at $4880 \mathrm{~nm}$ for $\mathrm{FAPbBr}_{3}$ film. (C) 2D-false color plot for fs-transient absorption in the mid-infrared regions for $\mathrm{FAPbl}_{3}$ film using $530 \mathrm{~nm}$ as an excitation source. (D) Extracted kinetic trace at $4960 \mathrm{~nm}$ for $\mathrm{FAPbl}_{3}$ film. 

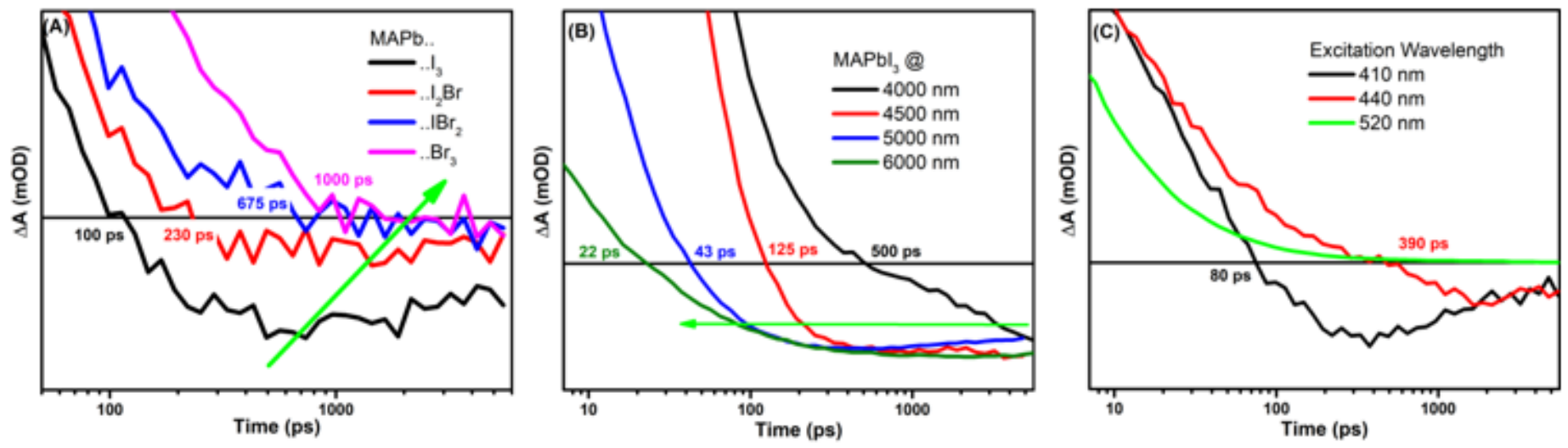

Figure 4

Dependence on chemical composition: (A) Normalized kinetic traces at $4900 \mathrm{~nm}$ extracted form $\mathrm{MAPbI}_{n} \mathrm{Br}_{3-\mathrm{n}}$ films using $520 \mathrm{~nm}$ as excitation showing the appearance of negative signals. Dependence on mid-IR detection window: (B) Normalized Kinetic traces for $\mathrm{MAPbl}_{3}$ film under excitation of $520 \mathrm{~nm}$ and at different probing wavelengths showing the appearance of negative signals. Dependence on excitation wavelengths: (C) Normalized kinetic traces for $\mathrm{MAPbl}_{3}$ film under various excitation wavelengths showing the appearance of negative signals.

\section{Supplementary Files}

This is a list of supplementary files associated with this preprint. Click to download.

- UltrafastTransientInfraredforProbingTrappingStatesinHybridPerovskiteFilmssubmissionSI.docx

- Scheme1.png 